\title{
Learning an Application of Superconductivity in Modern Mass Transportation
}

\author{
Jin Ping ${ }^{\dagger}$, Liu Hui* and Wan $\mathrm{Li}^{* *}$
}

\begin{abstract}
Applications of superconducting technology in transportation is more straightforward than others, such as magnetic levitation (maglev) trains. A maglev train is of high speed \& low power-consumption, environmental friendly and safe, accompanied by some drawbacks. This article will introduce the application of superconducting technology in the urban mass transport system, and the bottleneck of this application.
\end{abstract}

Keywords : Superconducting technology, Maglev, Mass transportation

\section{Introduction}

Superconductivity has been a mysterious phenomenon, and a superconductor has been viewed as a miraculous material. Scientists all over the world, however, have never stopped researches on superconductivity since the Dutch physicist Heike Kamerlingh Onnes first found the superconducting phenomenon in 1911. After the discovery of the first high-temperature superconductor (HTS) $\mathrm{LaBaCuO}$ by J. G. Bednorz and K. A. Mueller [1], scientists of IBM in Zurich, in 1986, the increase of superconducting critical temperatures has brought the researches a big step forwardly and triggered its unprecedented applications. Superconductivity has wide prospect of applications in the areas of mass transportation, optoelectronics, military, aviation and aerospace, etc.

\section{Superconducting Phenomena and Superconductors}

In 1911, Heike Kamerlingh Onnes in Leiden University found that the resistance of mercury suddenly disappeared when it was cooled to $-268.98^{\circ} \mathrm{C}$, and he further found

\footnotetext{
Corresponding author: Association for Science \& Technology, Chongqing University, Chongqing 400030, China.

E-mail : pjin@cqu.edu.cn

${ }^{*}$ Chongqing University, Chongqing 400030

** Chongqing Academy of Science and Technology, Chongqing 401123
}

that many other metals and alloys would lose their resistance when cooled to similar low temperatures. Because of its unique conduction capability, Onnes called it "superconducting state", which shocked the whole world. He was awarded the Nobel Prize in physics in 1913 for his investigations on the properties of matters at low temperatures which led, inter alia, to the production of liquid helium. A conductor in superconducting state was then named a superconductor. The phenomenon of resistivity disappearance under some specific low temperature was called zero-resistance effect. Current would not produce heat when flowing through a conductor without resistance, and thus a strong current would be formed in the conductor, thus generating a super-strong magnetic field [2].

Superconducting materials are characteristic of the zeroresistance effect, Josephson Effect and Meissner effect. The significance of these effects of superconductors was not less than the discovery of semiconductors. Some scientists even predicted that applications of superconductivity would lead to a revolution of new technology. Scientists all over the world worked diligently to investigate this miracle phenomenon, and made a lot of important breakthroughs. In China, American Chinese physicist, the chief scientist of Chongqing Academy of Science and Technology, China; and a physics professor of Southern University, USA; Dr. J. D. Fan and his co-worker Dr. Yuriy Malozovsky offered a unified theory of high- and lowtemperature superconductivity by applying the theory of 
strongly coupled many particles [3], which they developed in 1990's, to a layered two-dimensionally structured system of electrons. The approach and method to describe the highand low-temperature superconductivity were uniformly achieved. They have made a great breakthrough in the explanation of the main experiments concerning superconducting phenomena, and pioneered in theoretical studies.

\section{Introduction to Maglev Trains}

At present, the economy develops rapidly, and transportation has become one of the most important stanchions for the social and economic developments. With the increase of living standard, people tend to travel more and more, and the requirements for transports become higher and higher. It also becomes an urgent demand in the state economics, so Chinese government had developed various transport conveyances during the eleventh Five-Year Plan period. Chinese Academician of Science, He Zuoxiu, said that China should build a railway network with multiple speeds, and tried to expand the application of magnetic levitation technology based on the regular train with speed of $100 \mathrm{~km}$ per hour and express train with speed at $300 \mathrm{~km}$ per hour [4].

A maglev train is a high-tech transportation conveyance based on the attraction and repulsion of a magnetic filed. The train is equipped with electromagnets, and the electromagnetic coils are installed under the rails. When the current is switched on, the magnetic filed produced by the coils and that from the electromagnets on the train vehicle remain the same all the time [5], thus levitate the vehicle because of the repulsive force between two like poles. Electromagnetic coils are also installed on the two sides of the rails, and the alternating current turns the coils into electromagnets, which will produce the attractive force with the electromagnets on the train and drives the train forward. The repulsive force levitates the train and the attractive force drives the train along the rails without frictional forces. A maglev train gets rid of the problems of such as the rail adhesion limit, the noise and wearing which traditional trains possess. Instead, it has quick start/ stop capability and strong climbing capacity.

Regular conductor maglev trains (RCMT) are in general suitable for short-distance transportation and environment friendly, and have low energy-consumption, light vibration, and low noise, so, the prospects are very broad. RCMT can run at the speed about $350 \mathrm{~km}$ per hour. The maglev train is driven by electricity, and therefore avoids the pollution produced by coal or oil. This kind of trains usually passes over the ground and hills with 4.5 meter high elevated railway, which avoids the damage to the ecological environment. The maglev train moves along the rails, and is equipped with the fire equipment according to the standard of an airplane. The bottom of the train equipped with arms that embrace the rail tracks firmly, so that the train will not run off the rail tracks.

The power driving the train comes from the current on the two sides of the rail tracks, and magnitude of the current is the same in one area, so it is impossible for several trains to have different speeds or directions, which eliminate the possibility for head-on collisions or tail gating.

Although maglev train has so many advantages, Shanghai Maglev Train was the first commercial high-speed maglev line in the world, connecting Shanghai Pudong International Airport and downtown Shanghai. Testing tracks have been built by many countries such as Japan, Germany, etc. but the maglev train still has a long way to become a mass transportation conveyance as regular trains. The reason is that there are many aspects needing to be improved.

The first aspect concerns the safety. The maglev system needs electromagnetic force to levitate, guide and drive the train, so the safety of the train without electric power supply cannot be overlooked. Besides, the stability and reliability, when driving at high speed, need long-term running tests. Other issues are the technological challenges. Because the train needs to levitate at a specific height, it has very high requirements on the flatness of the rail tracks and roadbed subsidence. As the electric engineer building the Shanghai Maglev Train system said, all the cables along the guideway are ordinary aluminum high-voltage cables imported from Germany, and would produce very high electric voltage when the current is flowing through it. He told the relevant department to pay attention to the work safety along the guideway and enhance the protection of these cables to avoid accidents.

The second is the economic question. Money became another issue for maglev train application, even if all the technological difficulties were solved. The investment on the Shanghai Maglev Train system was 38 billion RMB (about 5 billion USD). Germany has planned to build two maglev systems: one of them is $36.8 \mathrm{~km}$ long and will cost about 1.6 billion Euros; another one is $78.9 \mathrm{~km}$ and the investment would be 3.2 billion Euros. In practice, the cost varies based on the topography, ground and designed transportation capability. But, $1 \mathrm{~km}$ maglev rails need at least 300 million RMB (46 M USD) investments, i.e. 3000 RMB (460 USD) is necessary for every $1 \mathrm{~cm}$.

An alternative is low-temperature superconductive maglev train (LTSMT) that is a maglev train with lowtemperature superconductors (LTS) being the coils to supply stronger electrical currents in order to produce stron- 
ger magnetic field needed for levitation and propulsion. Since it has to utilize very expensive liquid helium to realize superconductivity, the cost becomes much higher. Moreover, another question is the environmental concern brought by low-temperature superconducting magnetic levitation technology, which consumes more energy for levitation than regular conductor magnetic levitation. The cooling system is very heavy, and the strong magnetic field will influence on human and the environment. Researchers need to consider how to avoid this influence. The noise by the maglev train also needs to be put into consideration [6].

Overall, LTS maglev train has bright prospects. Once all the practical difficulties on technology, cost, and pollution could be solved on the basis of theoretical research, the prospects of superconducting technology would be very bright in urban transportation. But the solution of these difficulties needs more and more scientists' and engineers' diligent work.

\section{High-temperature Superconducting Technology will Lead to a Revolution of Mass Transportation}

However, there is a better way to solve the problems existing in LTSMT and RCMT due to the discovery of the high-temperature superconductors (HTS) in 1986. Based on the special unique property owned by an HTS, it is possible to design a HTS maglev train (HTSMT) system that exploits the property of anti-magnetism of an HTS (Meisner Effect) to levitate a vehicle without using the heavy and costly vehicle-mounted electromagnets. HTS bulks are installed at the bottom of the vehicle to be cooled below its transition temperature with cheap liquid nitrogen. The rails are made of permanent magnets to produce a magnetic field needed to levitate the vehicle without begging electromagnets. Moreover, levitation, guidance and stability of a vehicle are unified together by the properties of HTS itself, simplifying designs of structure, lightening the body of vehicles and saving great amount of funds in construction. Propulsion and braking system of an HTSMT are provided by a linear motor system that is set ip on the ground. It is obvious that the HTSMT is much simpler and ligher than RCMT and LTSMT. Correspondingly, safety of transportation becomes much better than ever.

In additionf"HTSMT can reach a much higher speed that is comparable with or even higher than an airplane when it runs in a low-pressure tube. Its high speed and ability of operating all-weather make it better than an airplane in long distance transportation. Without wheels and friction, it can save 30\% electric energy compared with the most advanced high speed trains. At the speed of $500 \mathrm{~km} / \mathrm{h}$, energy consumption per seat per kilometer is only $1 / 3$ to $1 /$ 2 that of a plane, $30 \%$ less than that of a car. The maintenance cost on a train and rails is reduced because of absence of wheels. Moreover, the noise is very low because there is no friction between the train and rails and the magnitude of the magnetic field is comparable with earth's magnetic field, much lower than that from household utility.

China's scientists and engineers headed by Jiasu Wang and Suyu Wang $[7,8]$ had designed and constructed their first HTS maglev train prototype of "century" in 2000. Through efforts for ten years the technologies in design and structure have become mature. It is possible to set up a test section for entertainments or tourism operated at lowspeed. Now, J. D. Fan and Jiasu Wang are working together to promote the implementation of a plan that is devoted to pave a section of rails for low-speed HTSMT for tourism and entertainments, which is an engineering testing railway of HTSMT on the one hand, and also a commercially operational facility, on the other hand. A success of this plan can enhance public awareness of the advantages of HTSMT encourage governments to invest more in this project. Moreover, it needs time to accumulate data and experience in HTSMT and make a on-site test of operating an HTSMT behicle at a speed higher than $100 \mathrm{~km}$ per hour. Only if this is done, the dream of a realistic HTSMT transport convenyance can become true.

\section{References}

1. J. G. Bednorz and K. A. Müller (1986). "Possible high $\mathrm{T}_{\mathrm{c}}$ superconductivity in the $\mathrm{BaLaCuO}$ system", Z. Physik, Vol. B64, No. 1, pp. 189-193.

2. Qi Bin; Magnetic Suspension Technology and Its Inventer -Emile Bachelet, Science Mosaic, 2002 (06)

3. J. D. Fan and Y. M. Malozovsky (2001). "A unified description of high- and low-temperature superconductivity," Physica C, pp. 364-365.

4. Xu Xiaomei and Zhu Sihong (2005). Magnetic Suspension Technology and its Engineering Application, Journal of Agricultural Mechanization Research (06).

5. Shao Ke (2006). The Maglev Train, Children's Science and Technology, (04).

6. Ge Yusen (1998), What is the advantage of magnetic suspension technology, Global Science \& Economy Outlook, (10).

7. Wang Jiasu, Wang Suyu, Zeng Youwen, Huang Haiyu, Luo Fang, Xu Zhipei, Tang Qixue, Lin Guobin, Zhang Cuifang, Ren Zhongyou, Zhao Guomin, Zhu Degui, Wang Shaohua, Jiang He, Zhu Min, Deng Changyan, Hu Pengfei, Li Chaoyong, Liu Fang, Lian Jisan, Wang Xiaorong, Wang Lianghui, Shen Xuming and Dong Xiaogang (2002). "The first man- 
Jin Ping, Liu Hui and Wan Li / IJR, 5(4), 148-151, 2012

loading high temperature superconducting maglev test vehicle in the world", Physica C, 378-381: 809-814.

8. Wang J.S., S.Y. Wang, Z.Y. Ren, H. Jiang, M. Zhu, X.R. Wang, X.M. Shen, H.H. Song (2003). "Experiment results of high temperature superconducting maglev vehicle," Physica C, 386: 431-437.

Received(December 4, 2012), Accepted(December 15, 2012) 